\title{
Internet resources for health and medicine
}

\author{
By Lee Hancock
}

\section{Information on cancer, AIDS, and other bealth issues}

工 nternet use by health workers, and those interested in health issues, is growing rapidly. The number of new resources has expanded dramatically in the past year. Interfaces such as gopher and the World Wide Web (WWW) are making the Internet less cryptic, thus attracting large numbers of new users. Adding to this "structuring" is the use of subject trees to categorize resources. This article examines these medical resources as they are categorized by disease.

\section{Non-disease-specific programs and documents}

- HEALTHLINE. The University of Montana's Healthline service offers topics of general health interest. It covers topics of physical and mental health including sexuality, drug and alcohol information, academic tips, and dietary facts. Access: URL: gopher://selway.umt.edu: $700 / 1$.

- NLM's PRACTICE GUIDELINES-HSTAT. The National Library of Medicine (NLM) offers a free electronic resource which provides access to the full-text of clinical practice guidelines developed under the auspices of the Agency for Health Care Policy and Research (AHCPR). HSTAT also includes other documents useful in health care decision-making: National Institutes of Health (NIH) Consensus Statements, NIH Technology Assessments, and the U.S Preventive Services Task Force Guide to Clinical Preventive Services. Access: Telnet: text.nlm.nih.gov or URL: http://www.nlm. nih.gov/, select NLM Online Information Services.

- MEDSEARCH AMERICA. This is the only national health care employment network on the Internet. Standard services to Healthcare Job Seekers are free of charge. Access: Gopher: Type $=1$, Name=MedSearch America, Path=, Host $=$ gopher.medsearch.com, Port $=9001$ or URL: gopher://gopher.medsearch.com:9001/1; e-mail: office@medsearch.com.

- THE VIRTUAL LIBRARY-MEDICINE. This is a comprehensive listing of WWW resources for biology and medicine. Access: World Wide Web, URL: http://golgi.harvard.edu/biopages/ medicine.htm.

- THE VIRTUAL HOSPITAL. The Virtual Hospital (VH) is a continuously updated medical multimedia database stored on computers and accessed through high speed networks 24 hours a day. The $\mathrm{VH}$ will provide invaluable patient care support and distance learning to practicing physicians. Access: World Wide Web, $U R L$ : http://indy.radiology.uiowa.edu.Virtual Hospital.html; e-mail: librarian@indy.radiology. uiowa.edu.

\section{Health resources categorized by disease}

Menu trees are the current trend in trying to organize the perceived chaos of the Internet. Disease descriptions, treatment protocols, nursing information, news sources, and drug information are currently available. An excellent overall disease categorization on a gopher server is the Yale Biomedical Gopher of Diseases and Disorders. The server offers articles and resources on AIDS, cancer, diabetes, digestive disorders, and more. Access: URL: gopher:// yaleinfo.yale.edu. 


\section{AIDS}

- AIDS RELATED INFORMATION-NIAID GOPHER. This contains AIDSNews, CDC National AIDS Clearinghouse, and much more. $A C$ cess: URL: gopher://odie.niaid.nih.gov/11.

- SOUTH EAST FLORIDA AIDS INFORMATION NETWORK. This database contains information on AIDS research organizations, and individuals working within these organizations located in southeastern Florida. Available information also includes educational and informational services on AIDS topics, health and social services, and research eligibility. This database was created with the help of the $\mathrm{Na}$ tional Library of Medicine. Access: telnet 129.171.78.1, login: library, select L on main menu, select 1 on next menu.

- AIDS ALERT FOR HEALTH CARE WORKERS. This is an index to journal articles and occasional papers concerning the occupational health and safety issues of health care workers providing care to AIDS patients. The Alert is annotated and compiled by Charlotte Broome of the Ryerson Polytechnical Institute's Education and Life Sciences Library. Issues of the Alert will appear three to four times per year. $A C$ cess: The Alert is distributed electronically by the Institute for AIDS Information; e-mail: libr8508@ryerson. Contact: Bob Jackson, librarian for education and life sciences.

- AIDS. A listserv mailing list for the Sci.Med.AIDS Newsgroup. This is a redistribution list for the Usenet newsgroup Sci.Med.AIDS. Mail to the list is automatically forwarded to the moderator team for the newsgroup. Subscribe: LISTSERV@WUVMD. WUSTL.EDU or LISTSERV@WUVMD. Contact. Moderator team at aids@cs.ucla.edu.

- AIDSNEWS. The AIDSNews Forum is used for the discussion of any issue relating to AIDS/ARC. AIDS Treatment News reports on experimental and alternative treatments, especially those available now. Subscribe: LISTSERV@RUTGERS.EDU or LISTSERV@ RUTVM1. Contact: Michael Smith; e-mail. msmith@umaecs.

\section{Amyotrophic lateral sclerosis (ALS)}

- ALS. This electronic mailing list has been set up to serve the worldwide ALS community. This includes ALS patients, ALS support/discussion groups, ALS clinics, ALS researchers, etc Others are welcome to join. THIS IS NOT A LISTSERV SETUP. Contact: Bob Broedel; e-mail: bro@huey.met.fsu.edu.
- ALS DIGEST-Lou Gehrig's Disease. This electronic publication covers all aspects of Amyotrophic Lateral Sclerosis (ALS) or Lou Gehrig's Disease. This includes ALS patients, patient supporters, physicians, support groups, research centers, etc. To subscribe, unsubscribe, or to contribute notes, send e-mail to: Bob Broedel at bro@huey.met.fsu.edu.

\section{Alzheimer's disease}

- ALZHEIMER. ALZHEIMER is an e-mail discussion group for patients, professional and family caregivers, researchers, public policy makers, students, and anyone with an interest in Alzheimer's or related dementing disorders in older adults. ALZHEIMER is intended to provide interested individuals from various perspectives an opportunity to share questions, answers, suggestions, and tips. Subscribe: majordomo@wubios.wustl.edu and in the BODY of the message send the command: subscribe ALZHEIMER (no name necessary). Contact: ALZHEIMER-owner@wubios.wustl.edu.

\section{Cancer}

- BREAST CANCER INFORMATION CLEARINGHOUSE. The Breast Cancer Information Clearinghouse is an Internet-accessible resource for breast cancer patients and their families. Information currently available includes patient education materials from the American Cancer Society and the National Cancer Institute, statistical information, and much more. Access: Gopher: nysernet.org or URL: http:// nysernet.org/. Contact: tmdamon@nysernet.org.

- BREAST-CANCER. This is an open discussion list for any issue relating to breast cancer. It is an unmoderated list open to researchers, physicians, patients, family and friends of patients, for the discussion of related issues. Subscribe: LISTSERV@MORGAN.UCS.MUN.CA. Contact: Jon G. Church; e-mail: jchurch@kean. ucs.mun.ca.

- CANCERNET-GUIDE TO CANCER TREATMENT. This is a quick and easy way to obtain, through electronic mail, cancer information from the National Cancer Institute (NCI). CancerNet lets you request information statements from the NCI's Physician Data Query database, fact sheets on various cancer topics from the NCl's Office of Cancer Communications, and citations and abstracts on selected topics from the CANCERLIT database. Selected information is also available in Spanish. There is no charge for the service unless your local com- 
puter center charges for use of e-mail. The CancerNet contents list changes at the beginning of each month as new statements and other information is included. Access: 1) Address your mail message to: cancernet@icicb.nci.nih.gov. If you are not on Internet, you may have to change the format of the address. Consult your systems manager for the correct address format. 2) In the body of the message: a) if you need the CancerNet contents list, enter "help" to receive the most current list (substitute the word "spanish" for "help" if you want the contents list in Spanish). For example: cancernet@icicb.nci.nih.gov, subject: help $<$ This is the body of the message $>$; b) if you have the CancerNet contents list and would like to request a particular statement or piece of information, enter the code from the contents list for the desired information. If you want more than one piece of information, enter the code for each piece of information desired on a separate line within the message. Note: Individual statements may exceed $100 \mathrm{~K}$ and some mail systems are limited in the size of the mail messages a user can receive. Please check your mail and storage capacity prior to submitting requests.

The information in CancerNet is also available on several gopher servers as well as a number of secondary distributor sites. To access a gopher server if you have gopher client software on your host computer or $\mathrm{PC}$, point to gopher.nih.gov. CancerNet can also be accessed via telnet: gopher.ncc.go.jp (160.190.10.1), using "gopher" as the logon and password (additional gopher public access sites can also be accessed via telnet). You may have to go through several menus and submenus to access CancerNet on a gopher server. For a complete listing of all gopher and secondary sites, request item $\mathrm{cn}-400030$ from CancerNet.

If you have any further questions, call: (301) 496-7403 or send an Internet message to Cheryl Burg, CancerNet project manager, at: cheryl@icicb.nci.nih.gov.

- ONCOLINK-U. OF PENN MULTIMEDIA ONCOLOGY RESOURCE. Oncolink is a WWW server and gopher server oriented to cancer. This resource is directed to physicians, health care personnel, social workers, patients and their supporters. Access: World Wide Web, URL: http://cancer.med.upenn.edu. Contact: E. Loren Buhle Jr.; e-mail: buhle@xrt.upenn.edu.

- CER-L. This electronic discussion list is a public group for the discussion of cancer-relat- ed topics. Subscribe: LISTSERV@WVNVM. WVNET.EDU or LISTSERV@WVNVM. Contact: Susan Rodman; e-mail: u0ac3@wvnvm.bitnet.

\section{Diabetes}

- DIABETES. This is an International Research Project on Diabetes discussion group. Subscribe. LISTSERV\%IRLEARN.bitnet@HEARN. nic.SURFnet.nl or LISTSERV@IRLEARN. Contact: Martin Wehlou; -mail: wehlou@fgen.rug.ac.be.

- DIABETIC. This forum is open to all users on this and any other node to aid diabetic persons in the exchange of views, problems, anxieties, and other aspects of their condition. As this is a public forum, all messages are subject to review by anyone who might request a copy. Subscribe: LISTSERV\%PCCVM.BITNET@ cmsa.BerKeywordsley.EDU or LISTSERV@ PCCVM. Contact: R. N. Hathhorn; e-mail: sysmaint@pccvm.

\section{Lyme disease}

- LYMENET-L. Subscribers to LymeNet-L receive the LymeNet Newsletter about twice a month. This publication provides readers with the latest research, treatment and political news about the lyme disease epidemic. Contact: Marc Gabriel, editor-in-chief, the LymeNet Neusletter; e-mail: mcg2@lehigh.edu.

\section{Multiple sclerosis}

- MSLIST-L. The multiple sclerosis discussion list. Subscribe: LISTSERV\%TECHNION.AC. IL@VM.TAU.AC.IL or LISTSERV@TECHNION.

\section{Conclusion}

There are also entry points to specialty-based medical knowledge on Internet. Computer departments, medical schools, and infomatics programs tend to develop information sources. Medical specialties are just beginning to take an interest in the Internet. An example is the Global Emergency Medicine Archives which includes an online emergency medical journal. Access: World Wide Web, URL: http:// herbst 7.his.ucsf.edu.

Thomas Jefferson University (TJU) and the previously mentioned Yale offer access to medical information by specialty and topic. These are good starting points for exploration of medical Internet resources and give a good sense of their scope. TJU offers gopher topic categorized medical knowledge. The gopher allows a hypertext access to worldwide servers relat(Internet cont. on page 605) 
dation to provide national leadership in educating information professionals through providing graduate fellowships, hiring faculty, and supporting pilot projects in creating and accessing information.

\section{The University of Texas at Austin's Harry}

Ransom Humanities Research Center has received $\$ 181,716$ from the National Endowment for the Humanities to catalog and create automated access to the Alfred A. Knopf, Inc. archive, an invaluable resource for the study of American publishing history, literature, and culture in the twentieth century.

\section{Williams College in Williamstown, Mas-} sachusetts, has been awarded a $\$ 100,000$ grant from the Charles E. Culpeper Foundation to support preservation initiatives. Funds will be used over a two-year period to construct and equip a small state-of-the-art conservation laboratory for the College Archives and Chapin Library of Rare Books. Additional funds will be used to survey the collections in order to select materials to be reformatted or conserved in the laboratory.

\section{Acquisitions}

\section{The Bert Corona Oral History Collection}

has been donated to the California Ethnic and Multicultural Archives at the University of California, Santa Barbara, as a joint gift of professor Mario T. Garcia and Bert Corona, a labor organizer in the Mexican-American community in the 1930s-1950s. The materials document Corona's life history as published in Garcia's Memories of Chicano History: The Life and Narrative of Bert Corona and includes extensive taped interviews with Corona along with transcriptions, documents, and photographs.

\section{Graham Greene's personal library of}

Victorian detective fiction has been acquired by the Robert W. Woodruff Library at Emory University. The collection, which was begun by Greene and Dorothy Glover in the last years of the Second World War, includes 613 works of mystery and detection dating from the earliest days of the genre up to the turn-of-the-century (Edgar Allen Poe through the early work of Arthur Conan Doyle).

\section{A large collection of special event man-} agement materials from two leaders in that field, Joe Jeff Goldblatt and Nancy Lynner, has been acquired by George Washington University's Gelman Library. The donation includes videotapes, audiocassettes, and documents, including the original manuscript of Goldblatt's pioneering book, Special Events: The Art and Science of Celebration.

\section{A collection of scholarly journals be-}

longing to Henry A. Fischel, a professor in the Department of Near Eastern Language and Literature and the Program of Jewish Studies, has been acquired by the Indiana University Libraries, Bloomington. The large collection includes monographs, pamphlets, and journals in Greek, Hebrew, Aramaic, German, French, and English, all concerning the impact of Hellenism on Near Eastern languages and literature.

The personal papers of A. C. Greene, noted journalist, historian, and radio and television commentator, have been acquired by the Special Collections Division of the University of Texas at Arlington Libraries. Among the papers are a 30-year collection of daily journals maintained by Greene, voluminous correspondence with various literary figures (including most Texas writers of the 1960-1990 period), much material about Greene's involvement with the Texas Institute of Letters, and historical materials on Texas railroads and industries.

\section{(Internet cont. from page 566)}

ed to topics such as AIDS, biology, cancer, diabetes, disability information, epidemiology, etc. Access: World Wide Web, URL: gopher:/ tjgopher.tju.edu/11/medical/bytopic or gopher: tjgopher.tju.edu.

Of course, it's beyond the scope of this article to list all health resources available on the Internet. There are over 300 listserv lists alone. Over the years this author has cataloged about $700 \mathrm{k}$ worth of resources in a document titled Internet/Bitnet Health Science Resources. It covers a vast array of listservs lists, newsgroups, epublications, databases, gophers, WWW servers, and much more. The document is available via anonymous ftp from ftp2.cc.ukans.edu in the directory pub/hmatrix as the file medlstxx.txt or .zip. The $\mathrm{xx}$ in the address is the date of the release and will change with updates. 